\title{
Meltblowing: I-basic physical mechanisms and threadline model
}

\author{
S. Sinha-Ray, ${ }^{1}$ A. L. Yarin, ${ }^{1,2, a)}$ and B. Pourdeyhimi ${ }^{3}$ \\ ${ }^{1}$ Department of Mechanical and Industrial Engineering, University of Illinois at Chicago, 842 W. Taylor St., \\ Chicago, Illinois 60607-7022, USA \\ ${ }^{2}$ Center for Smart Interfaces, Technische Universität Darmstadt, Petersenstr. 32, 64287 Darmstadt, \\ Germany \\ ${ }^{3} 3427$ The Nonwovens Institute, Box 8301, North Carolina State University, Raleigh, North Carolina 27695- \\ 8301, USA
}

(Received 17 March 2010; accepted 22 May 2010; published online 13 August 2010)

\begin{abstract}
The work aims at the experimental and theoretical study of the mechanism of meltblowing. Meltblowing is a popular method of producing polymer microfibers and nanofibers en masse in the form of nonwovens via aerodynamic blowing of polymer melt jets. However, its physical aspects are still not fully understood. The process involves a complex interplay of the aerodynamics of turbulent gas jets with strong elongational flows of polymer melts, none of them fully uncovered and explained. To evaluate the role of turbulent pulsations (produced by turbulent eddies in the gas jet) in meltblowing, we studied first a model experimental situation where solid flexible sewing threadlines were subjected to parallel high speed gas jet. After that a comprehensive theory of meltblowing is developed, which encompasses the effects of the distributed drag and lift forces, as well as turbulent pulsations acting on polymer jets, which undergo, as a result, severe bending instability leading to strong stretching and thinning. Linearized theory of bending perturbation propagation over threadlines and polymer jets in meltblowing is given and some successful comparisons with the experimental data are demonstrated. (C) 2010 American Institute of Physics. [doi:10.1063/1.3457891]
\end{abstract}

\section{INTRODUCTION}

In meltblowing the key flow element is a polymeric liquid jet stretched by a high speed gas flow. Meltblowing as a technological process for polymer microfiber and nanofiber production is relatively unexplored. Its fluid- and aerodynamics attracted some limited attention in the research literature (some references will be mentioned below). Therefore, its most important peculiarities have not been analyzed indepth yet. A brief survey of the recent works devoted to meltblowing reveals the following. Several important experimental works ${ }^{1,2}$ and references therein demonstrate that polymer jet configurations in meltblowing are extremely transient and nonstraight at already several centimeters from the hole exits in die nosepiece, the jet-jet interactions are significant and merging of neighboring jets is quite possible. Thermal regimes and polymer rheology have a strong effect. Also, turbulence of the surrounding gas flow can have a very significant effect on the final characteristics of the meltblown nonwovens. However, it is improbable that polymer jet characteristics in meltblowing are determined by turbulent eddies alone. Indeed, the results of the experiments ${ }^{1}$ show that the initial sections of the jets in meltblowing (of the order of 4 $\mathrm{cm}$ ) are straight, even though the turbulent pulsations are the strongest in the surrounding high-speed gas flow. The jet bending pattern is thus inconsistent with the one driven solely by turbulent eddies. Therefore, rheological behavior of polymeric liquid is expected to have a significant effect on jet dynamics. Also, the interaction with the surrounding gas flow cannot be reduced to only the effect of turbulent eddies,

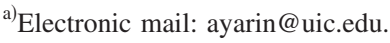

and the interaction with the mean flow can be very significant. In addition, a realistic description of the dynamics of bending perturbations should account for the interplay of the above-mentioned factors, which determine the pattern of bending perturbation propagation over polymer jets.

A number of defects in the meltblown nonwovens and drawbacks of the technology in general are rooted in the process fluid- and aerodynamics. Currently, the so-called roping (formation of streak-like structures in the webs and mats) is typically attributed to an uncontrolled turbulence. Such drawbacks of the process as the so-called fly (contamination of the surrounding gas by short and extremely thin fibers which do not connect to the fiber network) are generally attributed to too violent blowing conditions. These assumptions have, however, never been substantiated by either theoretical or experimental elucidation of the physical mechanisms of the process. The appearance of the so-called shots most probably results from the excessively high temperatures, when capillary breakup sets in (this situation is also insufficiently understood in the literature). The experimental data available in the literature are mostly descriptive and did not allow full recognition of the underlying physics and delineation of different effects responsible for the final parameters of meltblown nonwovens.

The theoretical works on meltblowing initially concentrated on the simplest model of a single straight jet. ${ }^{3}$ However, later on, the first steps were done in the direction of the quasi-one-dimensional equations of polymer nonstraight jets moving in gas, ${ }^{4,5}$ even though these works were seemingly unrelated to any preceding works on such jets discussed below, and did not result in a comparable model. Some prelimi- 
nary steps were taken to mimic turbulence. ${ }^{6}$ In a series of publications $^{7-10}$ the idea that stretching and bending of liquid jets in meltblowing is due exclusively to the turbulent pulsation field in the surrounding gas, which is responsible for the fluctuating gas drag force acting on the jet, was developed in full detail. Nevertheless, the aerodynamic effect of the distributed lift force was disregarded, and no realistic (viscoelastic) rheological behavior of polymeric liquids was accounted for in the meltblowing context.

Flapping flags and ropes in gas or water flows attracted some interest in fluid mechanics as an example of flowinduced vibrations of deterministic (nonturbulent) character. ${ }^{11-17}$ These works targeted a detailed deterministic description of flows about flapping ropes and flags, even including lateral forces resulting from shedding the von Karman vortex street in one case. In the other cases, the von Karman street-related forces were neglected as insignificant for the overall dynamics. All these works also accounted for the bending stiffness of flexible flags and ropes, even though in most cases it was relatively small in reality. This approach resulted in the fourth order PDE governing flag or rope dynamics, which does not allow an easy insight in the physical reasons of flapping, even though the obstruction comes from the higher order term which is negligible in many cases.

It was mostly overlooked that meltblowing is kindred to propagation of high speed liquid jets in gas environment, and the latter process has been understood in full detail in the 1980s. The works on high speed jets propagating in gas environment represent a good starting point for the study of meltblowing. Friction and heat transfer coefficients for straight cylindrical bodies (threadlines) moving in gas were measured experimentally and calculated theoretically in the framework of the boundary layer theory in numerous papers and monographs, ${ }^{18-20}$ which contain many empirical correlations and/or theoretical formulae for the corresponding friction coefficient and Nusselt number. They were successfully applied to calculate the drag force and heat flux on straight liquid jets moving in gas in the melt, dry or wet spinning processes. These correlations can be equally used locally in studies of meltblowing when the velocity determining drag and heat transfer should be understood as a relative velocity between rapidly moving gas and slower polymeric jet.

It is emphasized that the dynamics of liquid jets rapidly moving in gas is much richer than just propagation and thinning of straight jets characteristic of melt spinning, and so should be the dynamics of meltblowing. Indeed, the theoretical study of high speed liquid jets moving in gas began with the seminal works of Weber and Debye and Daen. ${ }^{21,22}$ In this case a rather complicated, three-dimensional (3D) problem on dynamic interaction of liquid jet and gas stream arises, where coupled evolution of the jet shape (which can become nonstraight) should also be determined. In Ref. 22 in the framework of the linear stability theory, it was shown that the inviscid liquid jets of circular cross-section are prone to planar bending if the jet velocity is sufficiently high. Such bending instability leads to an exponential growth of small bending perturbations. The instability is determined by a peculiar gas pressure distribution over the jet surface: gas pressure on the convex parts of the jet is lower than on the concave ones, which results in a distributed lateral lift force promoting further bending. The resulting distributed lift force is related rather to the potential (or mean) flow aerodynamics than to turbulent eddies and was not accounted for in Refs. 7-10 and several works on flag or rope flapping. The linear theory of Debye and Daen ${ }^{22}$ did not account for viscosity or viscoelasticity of liquid in the jet, possible large amplitudes of bending perturbations and their 3D (as in the experiments) rather than planar shape. It is practically impossible to address any of these factors in the framework of the Navier-Stokes equations or the corresponding 3D equations for the rheologically complex liquids due to a huge geometric complexity of the problem and complicating physical factors. That was the reason for the development of the general quasi-one-dimensional equations of liquid jets moving in gas in. $^{20-23}$ These equations were successfully used to describe the bending instability threshold and the whole nonlinear bending process of such jets including incorporation of the rheological effects and, in particular, viscoelasticity characteristic of polymer solutions and melts. In the present work this approach is applied to description of meltblowing and prediction of the characteristics of this process.

An additional process which is formally kindred to meltblowing is electrospinning of polymer nanofibers, where jet bending results from the electric Coulombic repulsion force. $^{24-26}$ The latter is proportional to the local curvature of the jet axis, which is similar to the distributed aerodynamic lift force. ${ }^{20,23}$

Meltblowing is an aerodynamically-driven process, in which polymer jets are accelerated and stretched by high speed gas streams (planar or axisymmetric, in general nonisothermal, subsonic turbulent jets). The aerodynamics of the mean flow in such jets is fairly well understood in the framework of the classical semiempirical turbulence models $^{27-29}$ and will be accounted for in the theoretical approach developed below.

The present work, the first of two (the accompanying part is in Ref. 30), is devoted to basic physical mechanisms responsible for meltblowing. To concentrate on the aerodynamic forces and the corresponding small (linearized) bending perturbations, a model situation of blowing of a solid, nonstretchable and flexible threadline is treated here. Meltblowing of polymer jet under isothermal and nonisothermal conditions with the account for polymer viscoelasticity and large (finite) perturbations is tackled in the accompanying paper. ${ }^{30}$ In the present paper Sec. II describes model experiments with solid flexible threadlines with the goal to elucidate the important physical aspects relevant in the context of meltblowing. In particular, we elucidate experimentally the peculiarities of turbulent fluctuations transmitted to the threadline where they result in visible bending perturbation. Propagation of these perturbations over the threadline is also studied in the experiments of Sec. II, which reveal a blow-up flapping region. A linearized theoretical model dealing with propagation of small bending perturbations over threadlines is given in Sec. III. Some comments on comparison of the theoretical predictions with the experimental data for threadlines are given in Sec. IV. Conclusions are drawn in Sec. V. 


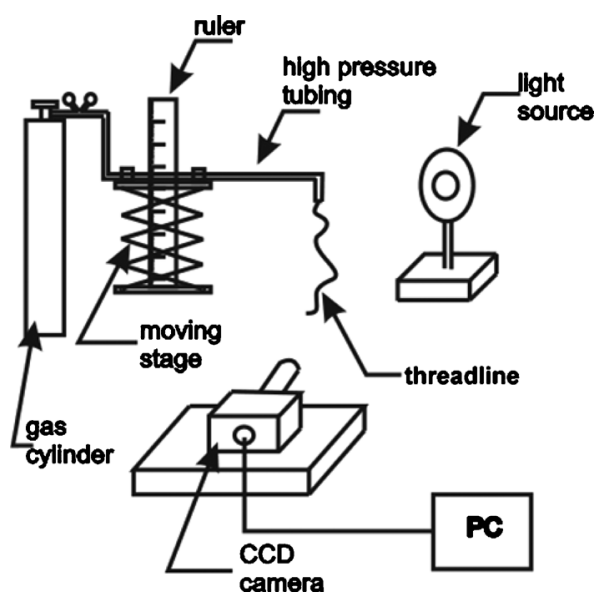

FIG. 1. Schematic of the experimental setup.

\section{EXPERIMENTS WITH FLEXIBLE THREADLINES}

A sketch of the experimental setup is shown in Fig. 1. It consists of a compressed gas (nitrogen at 25-40 bar) cylinder used to create blowing jet. High pressure tubing led to the nozzle (500 $\mu \mathrm{m}$ inner diameter) issuing the gas jet. A sewing threadline (mean diameter $110 \mu \mathrm{m}$, straightened by ironing before the experiments) was attached to the nozzle (Fig. 1). Blowing was directed downward and the nozzle positioning was adjusted using a moving stage with a ruler relative to the charge-coupled device (CCD) camera which was fixed (Fig. 1). A high speed CCD camera (MotionScopeRedlake Imaging Corporation) was used for high speed imaging. It was operated at $500 \mathrm{fps}$ using a shutter speed of $1 / 8500-1 / 10000 \mathrm{~s}$. When gas was blowing and the threadline was flapping, the high speed imaging was conducted for a number of segments of the threadline. At any new elevation of the threadline suspension point, a delay of 5 mins (sufficient for the transients fading) was adopted before data acquisition at that location. The images recorded with the high speed CCD camera were processed using the interface of MATLAB-R2007A. In addition, DSLR Nikon D70s camera was used at a very high shutter speed (1/4-1/6 s) to record the whole envelope of the flapping threadline at a single still image.

As a basic variant, a $\mathrm{L}=19.3 \mathrm{~cm}$-long threadline was used with gas blowing at $35 \mathrm{bar}(263.9 \mathrm{~m} / \mathrm{s}$-cf. the Appendix). The threadline was vigorously flapping under such conditions. The time series for the lateral threadline displacements at two locations are depicted in Fig. 2. Two specific positions were chosen for illustration. Figure 2(a) shows the evolution at $\mathrm{x}=10.4 \mathrm{~cm}$ and Fig. 2(b) shows the evolution at $\mathrm{x}=14.82 \mathrm{~cm}$. It is emphasized that the dc part of the lateral displacement caused by a slight uncontrolled tilt of the gas jet axis relative to the vertical was subtracted from the data in Fig. 2 using fast Fourier transformation (FFT) of the recorded signal.

FFT of the time series for the lateral displacements of the threadline corresponding to the time intervals of $3 \mathrm{~s}$ (two shorter sections of the data of about $1.5 \mathrm{~s}$ are shown in Fig. 2) was done using the corresponding MATLAB routine. The absolute values of the Fourier coefficients obtained are plotted against frequency in Figs. 3(a) and 3(b). It is seen that the spectral band covered was up to $250 \mathrm{~Hz}$, with the spectrum being continuous. It is emphasized that the recorded signal obtained from the CCD operated at $500 \mathrm{fps}$, as well as its processing with FFT involving frequencies up to $250 \mathrm{~Hz}$, precluded probing frequencies higher than those in Figs. 3(a) and 3(b) (up to $250 \mathrm{~Hz}$ ). However, a very significant and gradual decrease in the signal amplitudes for the highfrequency harmonics allows one to assume that there are no significant higher frequency modes. Indeed, Figs. 3(a) and 3(b) show that above the frequency of about $167 \mathrm{~Hz}$, the Fourier coefficients become negligible compared to those with frequencies less than $167 \mathrm{~Hz}$, and thus, the importance of the whole high-frequency range in the compound signal is expected to be small. This is illustrated in Figs. 3(c) and 3(d) where the Fourier series with a truncated spectrum with frequencies less than $167 \mathrm{~Hz}$ (and the cut-off of the spectral band with frequencies above $167 \mathrm{~Hz}$ ) and the corresponding Fourier coefficients from Figs. 3(a) and 3(b) are plotted versus experimental data. It is clearly seen that the experimental data are reproduced fairly well in spite of the truncated spectrum.

It is emphasized that the unperturbed threadline is vertical in the experimental setup of Fig. 1, i.e. potentially represents itself a kind of a pendulum. The eigenfrequency of the pendulum-like oscillations $\omega_{\mathrm{p}}=(2 \pi)^{-1} \sqrt{\mathrm{g} / \mathrm{L}}$, with $\mathrm{g}$ being
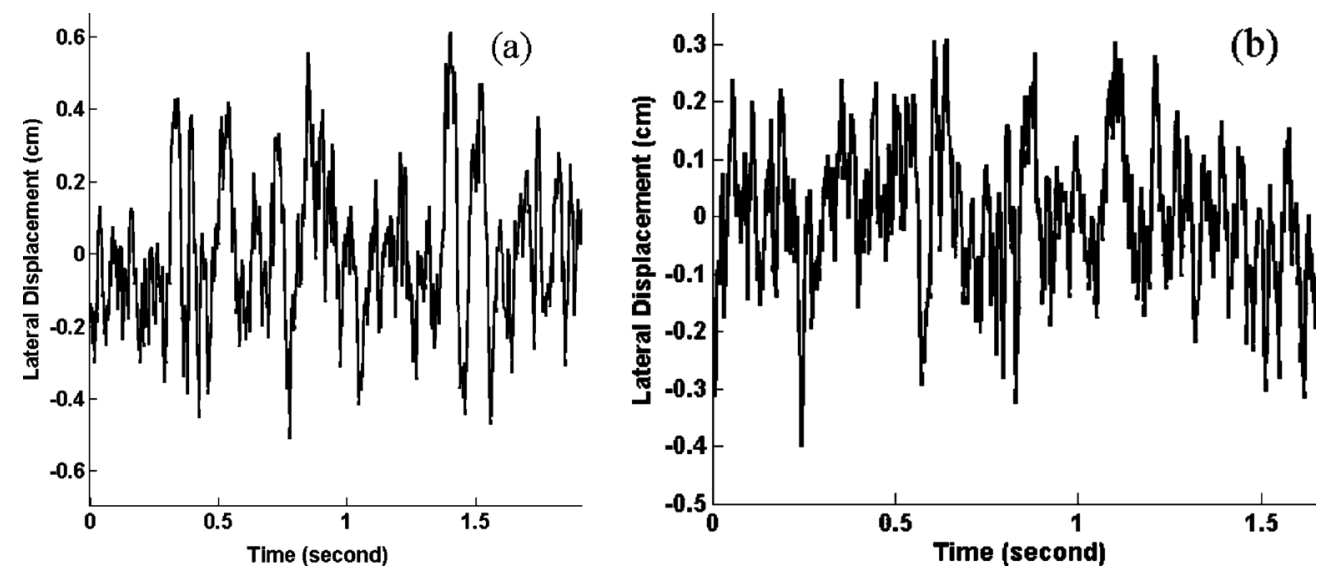

FIG. 2. Lateral displacements of the threadline at two locations: (a) $\mathrm{x}=10.4 \mathrm{~cm}$ and at (b) $\mathrm{x}=14.82 \mathrm{~cm}$ from the nozzle. 

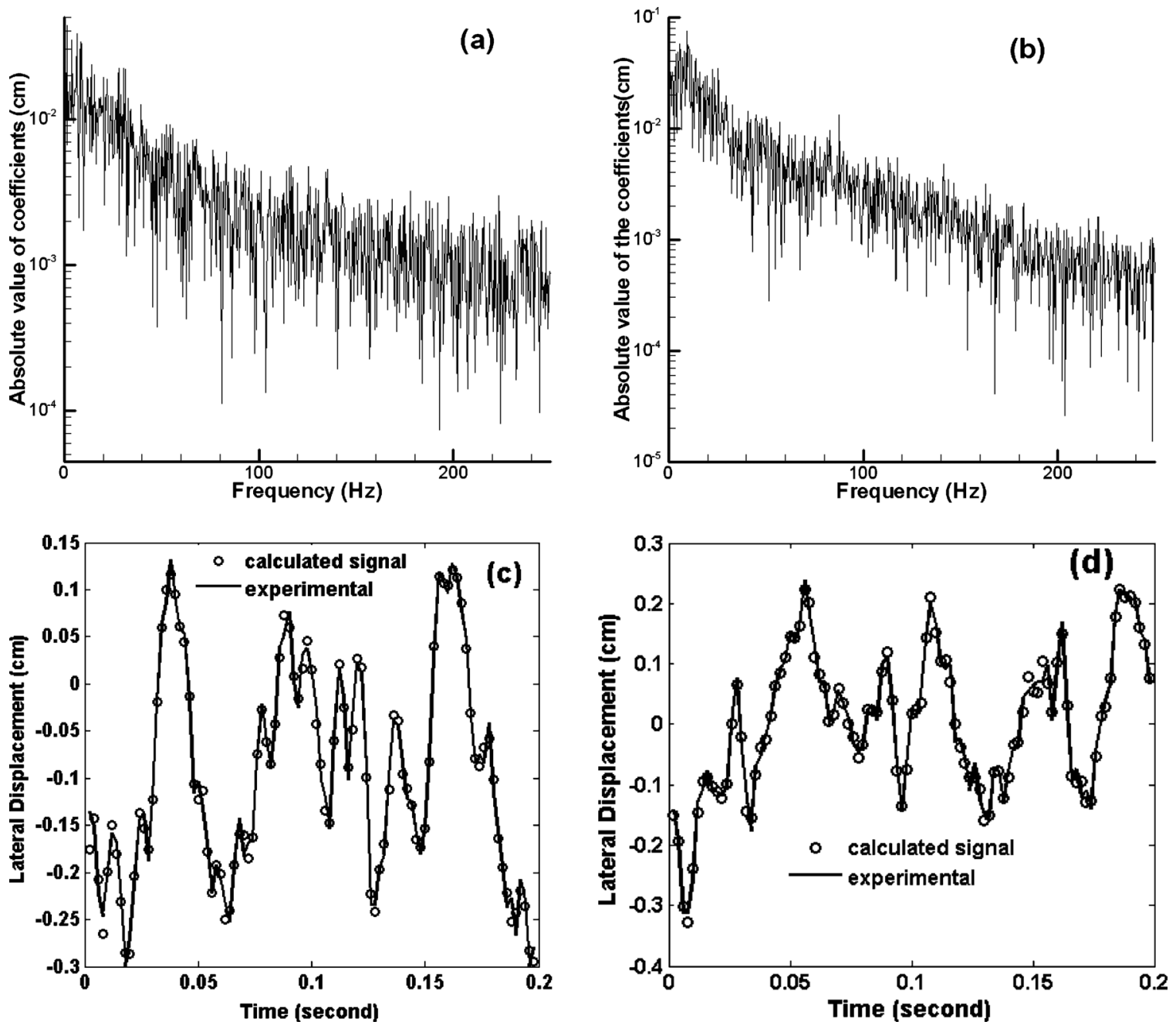

FIG. 3. Absolute values of the Fourier coefficients corresponding to the lateral displacement of the threadline at (a) $x=10.4 \mathrm{~cm}[$ Fig. $2(a)]$ and (b) $x$ $=14.82 \mathrm{~cm}$ [Fig. 2(b)]. (c) Lateral displacement at $\mathrm{x}=10.4 \mathrm{~cm}$ reconstructed using the Fourier series with the truncated spectrum. The calculated displacement is shown by symbols, the experimental data by solid line. (d) Same as (c) but at $x=14.82$.

gravity acceleration and the factor $(2 \pi)^{-1}$ included for comparison with the frequency in Figs. 3(a) and 3(b). Taking the pendulum length within the range $\mathrm{L} \approx 1-20 \mathrm{~cm}$ (since any part of the threadline can be excited), we find $\omega_{\mathrm{p}} \leq 5 \mathrm{~Hz}$. These are too low frequencies to cause resonances with the oscillations in Figs. 2 and 3, and thus can be excluded from consideration.

The character of the time series for lateral displacements of the threadline depicted in Fig. 2 can be also elucidated using the corresponding autocorrelation functions. The latter were calculated using standard MATLAB routine and are plotted in Fig. 4. It is clearly seen that the autocorrelation function rapidly decays and approaches zero-a clear sign of a chaotic process.

The threadline is subjected to turbulent perturbations of the surrounding turbulent gas jet. Denote by $\mathrm{U}_{\mathrm{g}}(0)$ the exit velocity of the gas jet, by $\mathrm{d}_{0}$ the nozzle diameter, and by $\nu_{\mathrm{g}}$ the kinematic viscosity of gas. Define the Reynolds number as $\operatorname{Re}_{\mathrm{d}}=\mathrm{U}_{\mathrm{g}}(0) \mathrm{d}_{0} / \nu_{\mathrm{g}}$. Then, the turbulence Taylor microscale in the axisymmetric gas jet according to Ref. 31 is equal to $\lambda=1.23 \mathrm{Re}_{\mathrm{d}}^{-1 / 2} \mathrm{x}$. Take for the estimate $\mathrm{U}_{\mathrm{g}}(0)=230 \mathrm{~m} / \mathrm{s}$ and $\mathrm{d}_{0}=0.05 \mathrm{~cm}$. Then, for air $\operatorname{Re}_{\mathrm{d}}=7667$. Therefore, at $\mathrm{x}$ $=1 \mathrm{~cm}$, turbulent eddy scales are of the order of $\lambda$
$=0.014 \mathrm{~cm}$, and at $\mathrm{x}=10 \mathrm{~cm}$, they are of the order of $\lambda$ $=0.14 \mathrm{~cm}$. The corresponding frequencies are of the order of $^{29} \omega=\mathrm{U}_{\mathrm{g}}(0) / \lambda$, which is $10^{5}-10^{6} \mathrm{~Hz}$, well above the frequency band characteristic of the threadline oscillations (Fig. 3 ). On the other hand, the large eddies in the system gas jet/threadline are of the order of $\mathrm{L}=10 \mathrm{~cm}$, and their frequencies $\omega=\mathrm{U}_{\mathrm{g}}(0) / \mathrm{L}$ are $10^{3} \mathrm{~Hz}$. This is still significantly higher than the frequency band in Fig. 3.

Local interactions of random, high-frequency (compared to those of the threadline) turbulent eddies in gas with massive threadline resembles those responsible for the Brownian motion. In the latter case, multiple simultaneous tiny impacts in different directions produce macroscopic displacement of massive (submicron) particles on time scales much larger than those of the individual impacts. In the turbulence context this idea was developed by Taylor. In particular, a lateral averaged displacement in time $t$ produced by turbulent eddies is ${ }^{31,32}\langle\mathrm{~A}\rangle=\left[2\left\langle\mathrm{v}^{\prime 2}\right\rangle \tau \mathrm{t}\right]^{1 / 2}$, where $\left\langle\mathrm{v}^{\prime 2}\right\rangle$ is the correlation of the lateral velocity pulsation and $\tau$ the characteristic time scale of large eddies. In turbulent jets all the pulsation velocity components, in particular, $\mathrm{v}^{\prime}$ (lateral) and $\mathrm{u}^{\prime}$ (longitudinal) are of the same order. Thus, $\left\langle\mathrm{v}^{\prime 2}\right\rangle=\left\langle\mathrm{u}^{\prime} \mathrm{v}^{\prime}\right\rangle$. The charac- 

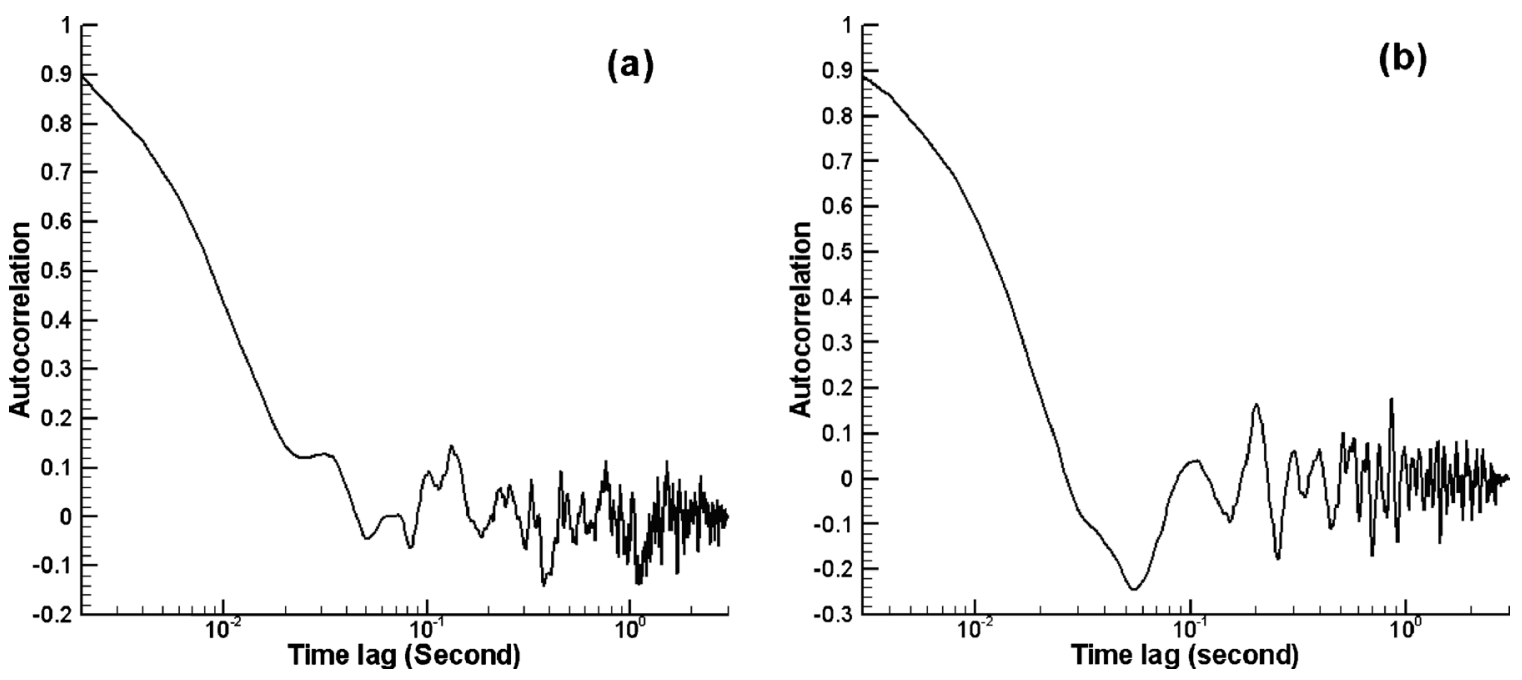

FIG. 4. Autocorrelation function for the time series for the lateral displacements of the threadline in Figs. 2(a) and 2(b) at (a) $x=10.4 \mathrm{~cm}$ and (b) $\mathrm{x}$ $=14.82 \mathrm{~cm}$.

teristic time scale of the large eddies is $\tau=(\partial \mathrm{u} / \partial \mathrm{y})^{-1}$, where $\mathrm{u}$ is the mean longitudinal velocity component, and $\mathrm{y}$ is the lateral coordinate. Therefore, $\left\langle\mathrm{v}^{\prime 2}\right\rangle \tau=\left\langle\mathrm{u}^{\prime} \mathrm{v}^{\prime}\right\rangle / \partial \mathrm{u} / \partial \mathrm{y}=\nu_{\mathrm{t}}$, where $\nu_{\mathrm{t}}$ is the kinematic eddy viscosity which plays here the role of the diffusion coefficient. In axisymmetric turbulent jets the kinematic eddy viscosity is constant over the jet and with a good accuracy is given by ${ }^{33} \nu_{\mathrm{t}}=0.015 \mathrm{U}_{\mathrm{g}}(0) \mathrm{d}_{0}$. Turbulent eddies can push a threadline element at a certain location only for the time of the order of the characteristic time $\tau_{\mathrm{r}}$ of propagation of a bending perturbation over the threadline (which plays the role of relaxation in this case). After that, the excursion leaves the previous location and propagates along the threadline. Therefore, we can estimate the amplitude of the threadline lateral displacements as $\left\langle\mathrm{A}_{\text {threadline }}\right\rangle$ $=\left(2 \nu_{\mathrm{t}} \tau_{\mathrm{r}}\right)^{1 / 2}$. The speed of propagation of bending perturbations along a stretched threadline is of the order of $[\mathrm{P} /(\mathrm{S} \rho)]^{1 / 2}$, where $\mathrm{P}$ is the tensile force in the threadline cross-section, $\mathrm{S}$ is the cross-sectional area $\left(\sigma_{\mathrm{xx}}=\mathrm{P} / \mathrm{S}\right.$ is the longitudinal stress in the threadline cross-section), and $\rho$ the threadline density. Therefore, the characteristic time of propagation of a bending perturbation over a threadline is $\tau_{\mathrm{r}}=\mathrm{L}[\mathrm{P} /(\mathrm{S} \rho)]^{-1 / 2}$. The tensile forces $\mathrm{P}$ is evaluated as $\mathrm{q}_{\tau} \mathrm{L}$ where $\mathrm{q}_{\tau}$ is the longitudinal air drag imposed on the unit length of the threadline. An appropriate expression for the drag force is given by Refs. 19 and 20 as

$$
\mathrm{q}_{\tau}=0.65 \pi \mathrm{a}_{0} \rho_{\mathrm{g}} \mathrm{U}_{0}^{2}\left(\frac{2 \mathrm{U}_{0} \mathrm{a}_{0}}{\nu_{\mathrm{g}}}\right)^{-0.81},
$$

where $\rho_{\mathrm{g}}$ and $\nu_{\mathrm{g}}$ are the gas density and kinematic viscosity, $\mathrm{U}_{0}$ is the gas velocity relative to the unperturbed threadline, and $\mathrm{a}_{0}=\mathrm{d}_{0} / 2$ is the cross-sectional radius of the threadline.

Taking for the estimate $\mathrm{L}=10 \mathrm{~cm}, \mathrm{~S}=\pi \mathrm{d}_{0}^{2} / 4=1.96$ $\times 10^{-3} \mathrm{~cm}^{2}$ (for $\left.\mathrm{d}_{0}=0.05 \mathrm{~cm}\right), \mathrm{U}_{0}=\mathrm{U}_{\mathrm{g}}(0)=230 \mathrm{~m} / \mathrm{s}$ and $\rho$ $=0.786 \mathrm{~g} / \mathrm{cm}^{3}$, we find for air $\nu_{\mathrm{t}}=0.015 \mathrm{U}_{\mathrm{g}}(0) \mathrm{d}_{0}$ $=17.25 \mathrm{~cm}^{2} / \mathrm{s}, \mathrm{q}_{\tau}=23.5 \mathrm{~g} / \mathrm{s}^{2}$, and $\tau_{\mathrm{r}}=0.0256 \mathrm{~s}$ (the value is in a very good agreement with the main threadline frequencies in Fig. 3, which are of the order of $\tau_{\mathrm{r}}^{-1}=39 \mathrm{~Hz}$ ). Therefore, $\left\langle\mathrm{A}_{\text {threadline }}\right\rangle=0.94 \mathrm{~cm}$, in a reasonable agreement with the experimental data in Fig. 2. The result confirms that the lateral threadline oscillations are imposed by multiple impacts of large turbulent eddies and restricted by propagation of bending perturbations along the threadline.

Another parameter of interest is the length of the threadline where flapping is the most vigorous. This requests a definition of vigorous flapping in the experiment. Two different definitions and methods based on them were used in the present work. In the first one, the images of the free-end part of the threadline were analyzed using high speed CCD images. The threadline cross-sections from which large lateral displacements of the free-end part were visible are denoted by arrows in the images (Fig. 5).

The flapping region depicted in Fig. 5 shows that propagation of bending perturbations along the threadline serves as a relaxation mechanism for lateral oscillations imposed by turbulent eddies only in the strongly stretched part at the beginning of the threadline, which supports practically the whole air drag force. On the other hand, the free end of the threadline is almost unloaded and imposes weak restrictions on the lateral excursions driven by turbulent eddies. Moreover, bending perturbations entering there from the upper part of the threadline can amplify with practically no restriction, delivering their whole energy parcel into such lateral motion. That is one of the reasons of the strong amplification of the bending perturbations of the threadline close to the free end, which results in flapping. Another reason for strong amplification will be in the appearance of the distributed lift force, which also diminishes the restricting effect of the threadline tension and is discussed in detail in Sec. III.

Another method of determining a vigorously flapping part of the threadline was based on the threadline envelope photographed by DSLR Nikon D-70s camera (Fig. 6). This method is capable of observations of almost the whole threadline length (in distinction from the first method based on CCD images), which allows an easy recognition of the flapping part of the threadline (Fig. 6).

Comparison between the results of the two methods of measuring the flapping part revealed the following. In the experiment with a $18.5 \mathrm{~cm}$ long threadline subjected to 

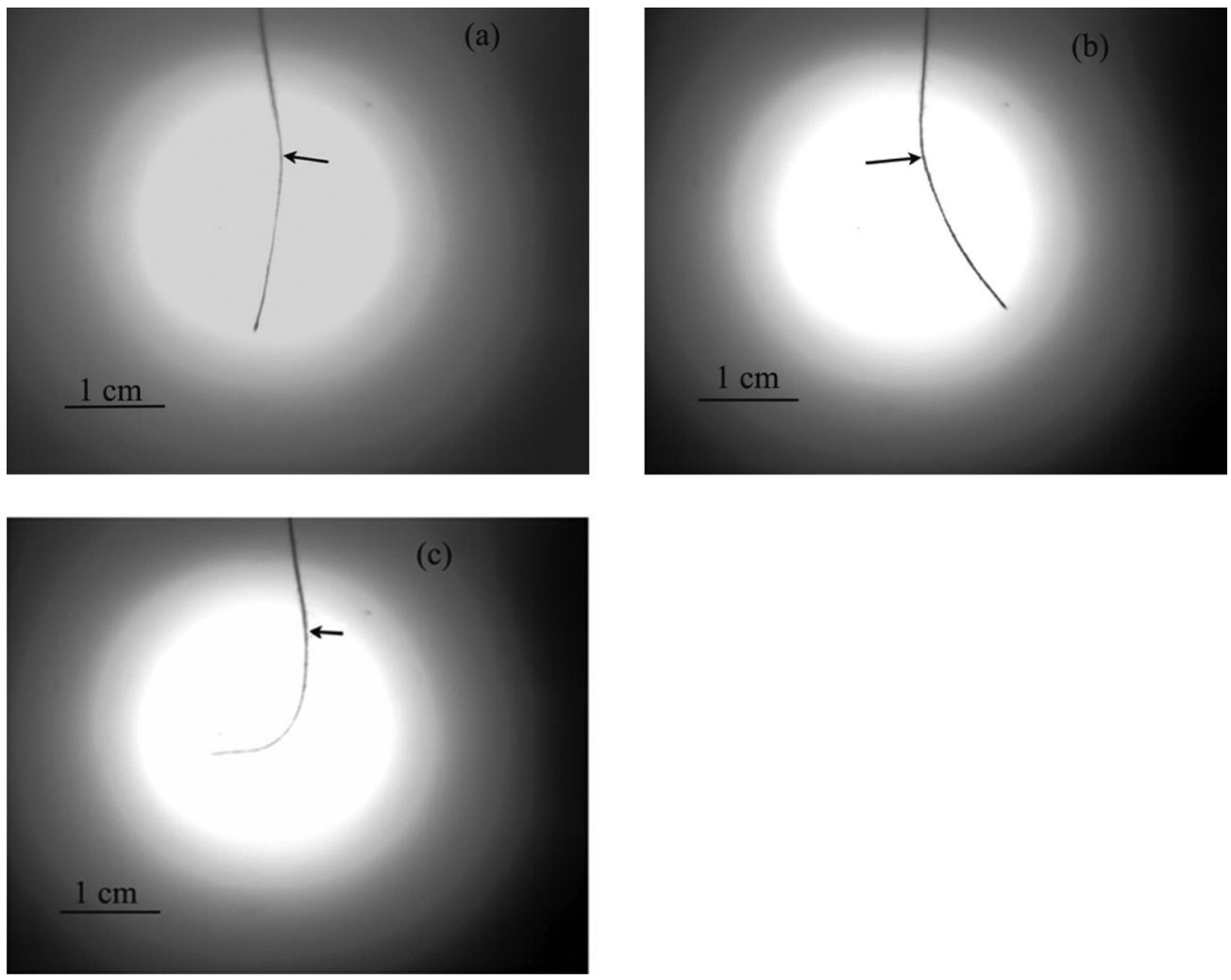

FIG. 5. Flapping of a $19.3 \mathrm{~cm}$ long threadline, which was subjected to gas jet issued at 35 bar recorded by CCD. The arrows show the beginning of the flapping part of the threadline. (a)-(c): Three different snapshots from the same experiment are shown to illustrate the different configurations recorded.

blowing at 35 bar $(263.9 \mathrm{~m} / \mathrm{s})$, the flapping length found averaging the data recorded by CCD camera was $1.82 \mathrm{~cm}$. On the other hand, the application of the method of Fig. 6 based on the data recorded by DSLR camera revealed the length of the flapping end of the threadline as $1.76 \mathrm{~cm}$. Therefore, the results obtained by the two different methods are sufficiently close. The slight difference in the values of the flapping length found by the two methods can be attributed to the following reasons: (i) the pixel resolution of the images obtained by DSLR camera is not sharp enough; (ii) due to the 3D nature of flapping, the actual beginning crosssection of the flapping part might be off by a few pixels from

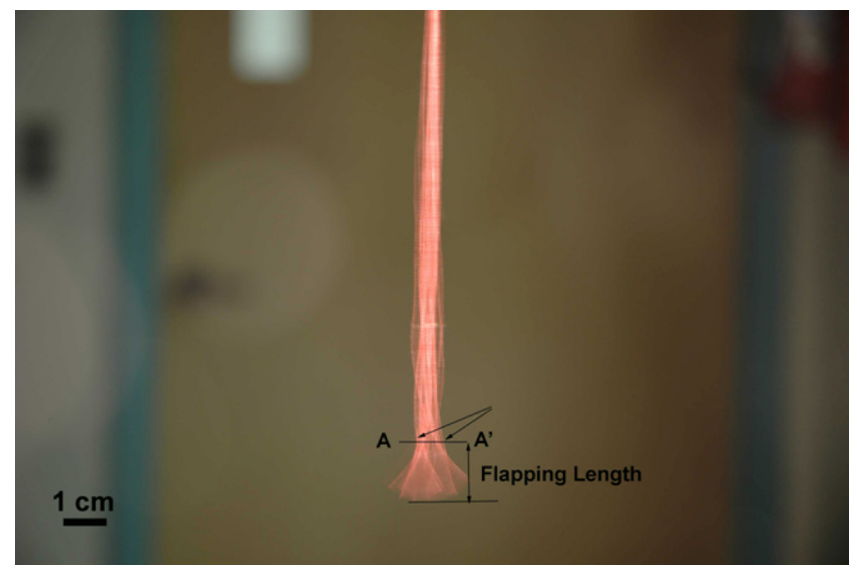

FIG. 6. (Color online) Determination of the flapping part of the threadline. The beginning of the flapping section is indicated by the inclined arrows. Experiment with a $19 \mathrm{~cm}$ long threadline subjected to gas jet issued at 28 bar. the one in the images; (iii) in spite of the fact that the threadlines were ironed and straightened prior to the observations, they always possessed some local nonzero curvatures along their length; and (iv) a certain inhomogeneity of the threadlines.

The method illustrated in Fig. 6 was applied to determine flapping lengths of threadlines of different lengths subjected to the same blowing speed. The experiment began with a $21 \mathrm{~cm}$ long threadline subjected to parallel gas jet flow issued at 35 bar $(263.9 \mathrm{~m} / \mathrm{s})$. After the transient effects had faded, the corresponding flapping length was measured as in Fig. 6. Then, blowing was ceased and the threadline was cut shorter. Then, blowing was turned on once again and the corresponding flapping length was measured. After that, the procedure was repeated again and again until the threadline length of $10.1 \mathrm{~cm}$. The results are shown in Fig. 7(a). It can be seen that the experimental points are clearly subdivided into two groups-one for shorter threadlines (10 to 16 $\mathrm{cm}$ long) and the other one-for the longer ones (16 to $21 \mathrm{~cm}$ long). In the "shorter" group the data suggest some variation in the flapping length $\mathrm{L}_{\mathrm{f}}$ versus threadline length $\mathrm{L}$, albeit it might be caused by data scatter. Note that the shorter threadlines might be significantly affected by such end effects as shedding vortices forming the von Karman street, as it was suggested in Ref. 15. On the other hand, in the "longer" group the flapping length is practically independent of the threadline length L.

Using the well-known analytical solution for the axisymmetric turbulent gas jets, ${ }^{28,33}$ it is easy to evaluate the ratio $\mathrm{u}_{\mathrm{gA}} / \mathrm{U}_{\mathrm{g}}(\mathrm{x})$ where $\mathrm{u}_{\mathrm{gA}}$ is the gas velocity value at points $\mathrm{A}$ and 

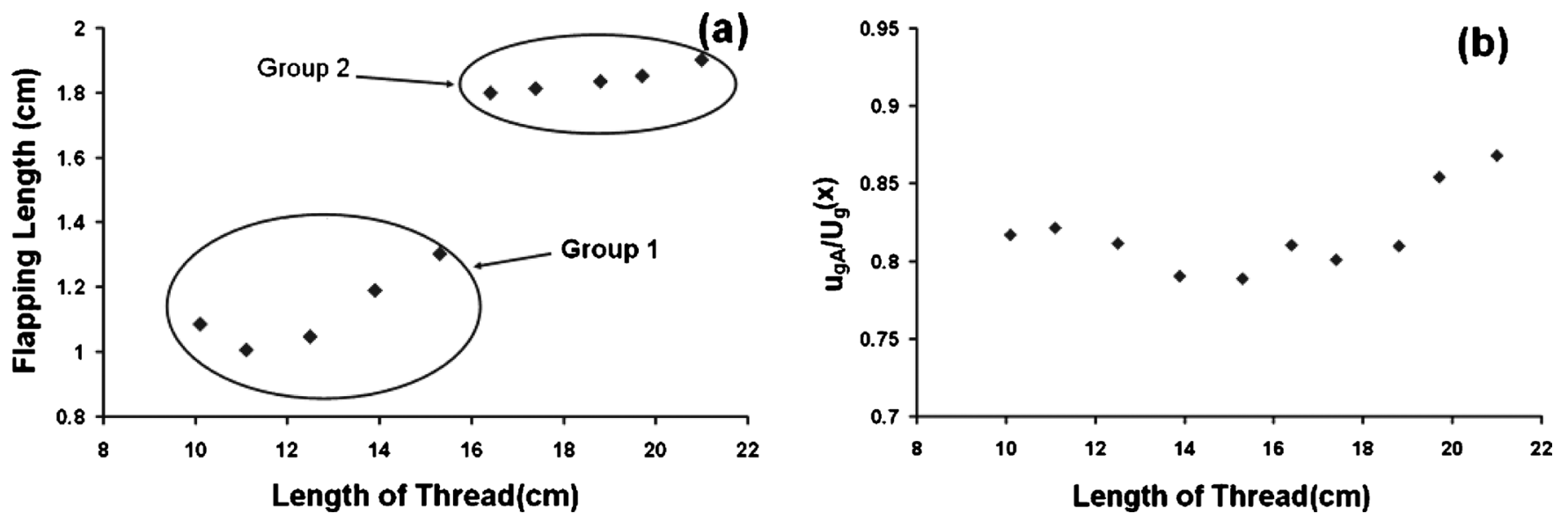

FIG. 7. (a) Flapping length vs threadline length. (b) Gas velocity at points A and $A^{\prime}$ in Fig. 6 relative to the local axial velocity value $\mathrm{U}_{\mathrm{g}}(\mathrm{x})$ for different threadlines.

$\mathrm{A}^{\prime}$ in Fig. 6, and $\mathrm{U}_{\mathrm{g}}(\mathrm{x})$ is the local axial velocity of the gas jet. The results are shown in Fig. 7(b). They demonstrate that the part of the threadline preceding the flapping part is located within the core of the surrounding gas jet corresponding to the range of $\mathrm{u}_{\mathrm{gA}} / \mathrm{U}_{\mathrm{g}}(\mathrm{x})$ from 0.78 to 0.87 , i.e., to the gas velocity values of $78 \%-87 \%$ of the local axial gas jet velocity in cross-section $\mathrm{AA}^{\prime}$.

In an additional experiment a $19 \mathrm{~cm}$ long threadline was subjected to gas jets issued at different pressures from 24 to 40 bar with a step of 2 bar (blowing velocities $\mathrm{U}_{\mathrm{g}}(0)$ $=181.6-274.1 \mathrm{~m} / \mathrm{s}$, cf. the Appendix) and the flapping length was measured using the method of Fig. 6 . The results are depicted in Fig. 8(a) in logarithmic scales. They reveal the scaling $\mathrm{L}_{\mathrm{f}} \sim \mathrm{U}_{\mathrm{g}}(0)$ at $\mathrm{U}_{\mathrm{g}}(0) \leq 230 \mathrm{~m} / \mathrm{s}$. At $\mathrm{U}_{\mathrm{g}}(0)$ $>230 \mathrm{~m} / \mathrm{s}$ the scaling changes to $\mathrm{L}_{\mathrm{f}} \sim \mathrm{U}_{\mathrm{g}}(0)^{2.42}$. The values of the ratio $\mathrm{u}_{\mathrm{gA}} / \mathrm{U}_{\mathrm{g}}(\mathrm{x})$ in the cross-sections corresponding to points $A$ and $A^{\prime}$ in Fig. 6 for all different blowing velocities are shown in Fig. 8(b). They demonstrate that the part of the threadline part preceding the flapping part is located within the core of the surrounding gas jet corresponding to the range of $\mathrm{u}_{\mathrm{gA}} / \mathrm{U}_{\mathrm{g}}(\mathrm{x})$ from 0.75 to 0.95 , i.e., to the gas velocity values of $75 \%-95 \%$ of the local axial gas jet velocity in cross-section $\mathrm{AA}^{\prime}$.

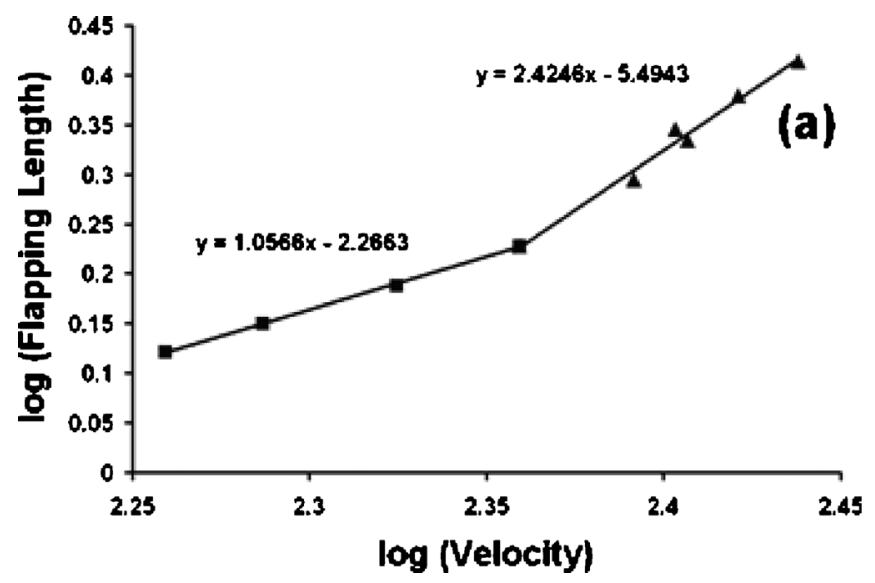

\section{THEORETICAL: SOLID FLEXIBLE THREADLINE IN PARALLEL HIGH SPEED GAS FLOW}

Consider a solid flexible threadline subjected to parallel high speed gas flow. The threadline is assumed to be nonstretchable and with negligible bending stiffness. Its crosssectional radius $\mathrm{a}_{0}$ is constant. In the unperturbed state the threadline is straight and aligned in the flow direction and does not move. The force balance in the threadline in this state reads

$$
\frac{\mathrm{dP}}{\mathrm{dx}}+\mathrm{q}_{\tau}=0
$$

where $\mathrm{P}=\sigma_{\mathrm{xx}} \pi \mathrm{a}_{0}^{2}$ is the longitudinal force acting in the threadline cross-section (with $\sigma_{\mathrm{xx}}$ being the normal stress), and $\mathrm{q}_{\tau}$ is the distributed aerodynamic drag force acting on a unit length of the threadline. An appropriate expression for the drag force is given by Eq. (1) in Sec. II. The longitudinal axis $\mathrm{Ox}$ is directed along the unperturbed threadline, with $\mathrm{x}=0$ corresponding to a certain "initial" cross-section which is discussed in detail below.

The normal stresses in the threadline cross-sections are of the elastic origin. Since the threadline stretchability is assumed to be very low (which means that its Young's modulus

FIG. 8. (a) Flapping length vs the gas jet velocity at the nozzle exit. (b) Gas velocity at points A and $\mathrm{A}^{\prime}$ in Fig. 6 relative to the local axial velocity value $\mathrm{U}_{\mathrm{g}}(\mathrm{x})$ for different blowing velocities. 


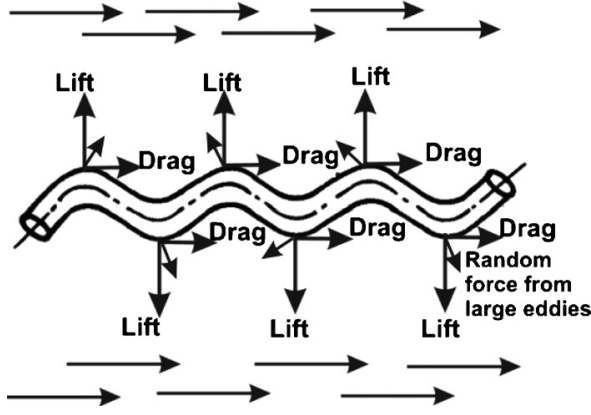

FIG. 9. Distributed forces acting on perturbed threadlines (and polymer jets) subjected to high speed gas flow.

is sufficiently high), the static (and, further on, dynamic) threadline elongation is assumed to be negligible, even though the stress $\sigma_{\mathrm{xx}}$ might be large.

The threadline length is $\mathrm{L}$, and at the free end $\mathrm{x}=\mathrm{L}$ the normal stress vanishes, i.e., $\left.\sigma_{\mathrm{xx}}\right|_{\mathrm{x}=\mathrm{L}}=0$. Then, Eq. (2) is integrated to yield the normal stress distribution along the unperturbed threadline

$$
\sigma_{\mathrm{xx}}=\frac{\mathrm{q}_{\tau}(\mathrm{L}-\mathrm{x})}{\pi \mathrm{a}_{0}^{2}} .
$$

In particular, the largest stress is achieved in the "initial" cross-section of the threadline at $\mathrm{x}=0$, which is $\sigma_{\mathrm{xx} 0}$ $=\sigma_{\mathrm{xx}}(0)=\mathrm{q}_{\tau} \mathrm{L} /\left(\pi \mathrm{a}_{0}^{2}\right)$, since it supports practically the whole aerodynamic drag applied to the threadline.

The solid flexible threadline is subjected to bending perturbations, which can result in lateral threadline motions due to the appearance of the normal component $\mathrm{q}_{\mathrm{n}}$ of the aerodynamic force per unit threadline length related to the mean flow. $^{20-23}$ This component is of the inviscid origin and resembles a distributed lift force, which originates from the unsteady Bernoulli equation for gas motion. Obviously, it is not alone, since the unsteady Bernoulli equation in addition produces the force component associated with the added mass. However, in high speed flows the lift force is the dominant one compared to the added mass effects, since only it is proportional to $\mathrm{U}_{0}^{2}$, whereas the additional force components are only linear in $\mathrm{U}_{0}$. It is emphasized, that we do not include here the effect of the turbulent pulsations in the gas flow, which will be accounted for separately.

As it is shown in Refs. 20-23, the distributed lift force is proportional to the local curvature of the threadline axis, and in the linear approximation (for small bending perturbations) is given by

$$
\mathrm{q}_{\mathrm{n}}=-\rho_{\mathrm{g}} \mathrm{U}_{0}^{2} \pi \mathrm{a}_{0}^{2} \frac{\partial^{2} \mathrm{H}}{\partial \mathrm{x}^{2}},
$$

where $\mathrm{H}=\mathrm{H}(\mathrm{x}, \mathrm{t})$ represents the curved threadline axis. Note that planar bending is considered here for simplicity, whereas the generalization to the $3 \mathrm{D}$ bending is straightforward. ${ }^{20,23}$ The distributed drag and lift forces acting on a perturbed threadline subjected to gas flow are shown schematically in Fig. 9. Random force imposed by turbulent pulsations is also shown in Fig. 9 for completeness.

The linearized lateral momentum balance for the perturbed threadline in a high speed gas flow reads

$$
\rho \pi \mathrm{a}_{0}^{2} \frac{\partial \mathrm{V}_{\mathrm{n}}}{\partial \mathrm{t}}=\mathrm{kP}+\mathrm{q}_{\mathrm{n}}
$$

where $\rho$ is the threadline density, $\mathrm{V}_{\mathrm{n}}=\partial \mathrm{H} / \partial \mathrm{t}$ is the lateral local velocity of the threadline, and $\mathrm{k}=\partial^{2} \mathrm{H} / \partial \mathrm{x}^{2}$ is the local curvature.

Accounting for Eq. (4), Eq. (5) takes the following form:

$$
\frac{\partial^{2} \mathrm{H}}{\partial \mathrm{t}^{2}}+\frac{\left[\rho_{\mathrm{g}} \mathrm{U}_{0}^{2}-\sigma_{\mathrm{xx}}(\mathrm{x})\right]}{\rho} \frac{\partial^{2} \mathrm{H}}{\partial \mathrm{x}^{2}}=0,
$$

where $\sigma_{\mathrm{xx}}(\mathrm{x})$ is given by Eq. (3).

Equation (6) is rather peculiar. It shows that in regards to the lateral motion of the threadline, the distributed lift force diminishes the effect of stretching imposed by the aerodynamic drag. Correspondingly, if $\sigma_{\mathrm{xx} 0}>\rho_{\mathrm{g}} \mathrm{U}_{0}^{2}$, this equation is hyperbolic at $0 \leq \mathrm{x} \leq \mathrm{X}_{*}$ where $\sigma_{\mathrm{xx}}>\rho_{\mathrm{g}} \mathrm{U}_{0}^{2}$, and elliptic at $\mathrm{x}_{*} \leq \mathrm{x} \leq \mathrm{L}$ where $\sigma_{\mathrm{xx}}<\rho_{\mathrm{g}} \mathrm{U}_{0}^{2}$, since according to Eq. (3) the normal stress decreases along the threadline. The transition cross-section $\mathrm{x}_{*}$ according to Eq. (3) is determined by the condition

$$
\rho_{\mathrm{g}} \mathrm{U}_{0}^{2}-\frac{\mathrm{q}_{\tau}\left(\mathrm{L}-\mathrm{X}_{*}\right)}{\pi \mathrm{a}_{0}^{2}}=0,
$$

which yields the length of the "elliptic" (flapping) part of the threadline $\mathrm{L}_{\mathrm{f}}$ as

$$
\mathrm{L}_{\mathrm{f}}=\mathrm{L}-\mathrm{x}_{*}=\frac{\mathrm{a}_{0}}{0.65}\left(\frac{2 \mathrm{U}_{0} \mathrm{a}_{0}}{\nu_{\mathrm{g}}}\right)^{0.81} .
$$

Equation (8) shows that the length of the "elliptic" part of the threadline does not depend on its length L. On the other hand, it increases with blowing speed as $\mathrm{U}_{0}^{0.81}$.

The fact that Eq. (6) changes type at $\mathrm{x}=\mathrm{x}_{*}$ makes it kindred to the Tricomi equation, albeit different from it.

The general solution of Eq. (6) with $\sigma_{\mathrm{xx}}$ given by Eq. (3) is readily found by the method of characteristics as

$$
\begin{aligned}
\mathrm{H}(\mathrm{x}, \mathrm{t})= & \Phi\left[\int_{0}^{x} \frac{\mathrm{dx}}{\left[\mathrm{q}_{\tau}(\mathrm{L}-\mathrm{x}) / \pi \mathrm{a}_{0}^{2}-\rho_{\mathrm{g}} \mathrm{U}_{0}^{2}\right] / \rho}-\mathrm{t}\right] \\
& +\mathrm{F}\left[-\int_{0}^{x} \frac{\mathrm{dx}}{\left[\mathrm{q}_{\tau}(\mathrm{L}-\mathrm{x}) / \pi \mathrm{a}_{0}^{2}-\rho_{\mathrm{g}} \mathrm{U}_{0}^{2}\right] / \rho}-\mathrm{t}\right],
\end{aligned}
$$

where $\Phi(\cdot)$ and $\mathrm{F}(\cdot)$ are arbitrary functions.

Perturbations on the threadline are imposed by turbulent pulsations in the surrounding turbulent gas jet. The experimental data discussed in Sec. II show that significant perturbations of the threadline configuration are visible at some distance from the suspended end of the threadline. In the first approximation, it is assumed that at a certain relatively small distance from the suspended end perturbations of frequencies and amplitudes corresponding to the local turbulent pulsation structure of the mixing layer in the gas jet are imposed on the threadline, whereas down the threadline no new perturbations are imposed, since the gas jet widens. This crosssection of the threadline where bending perturbations are imposed is considered to be the "initial" one, and $x=0$ there. In this cross-section it is assumed that the threadline is subjected to small lateral perturbations generated in the mixing layer of the gas jet, so that 


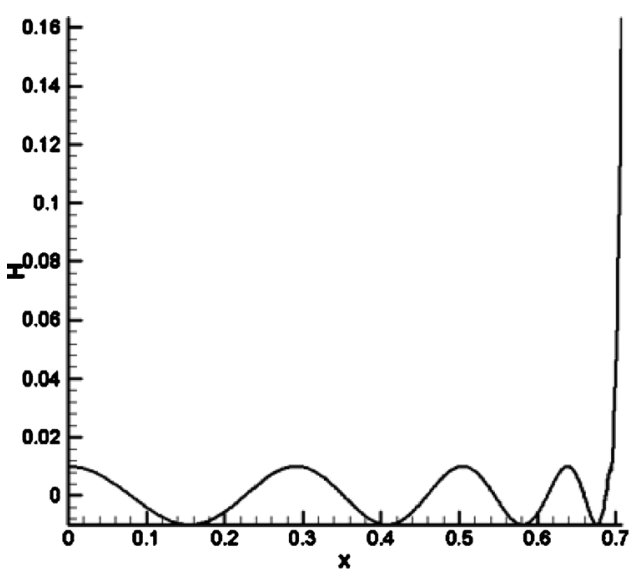

(a)

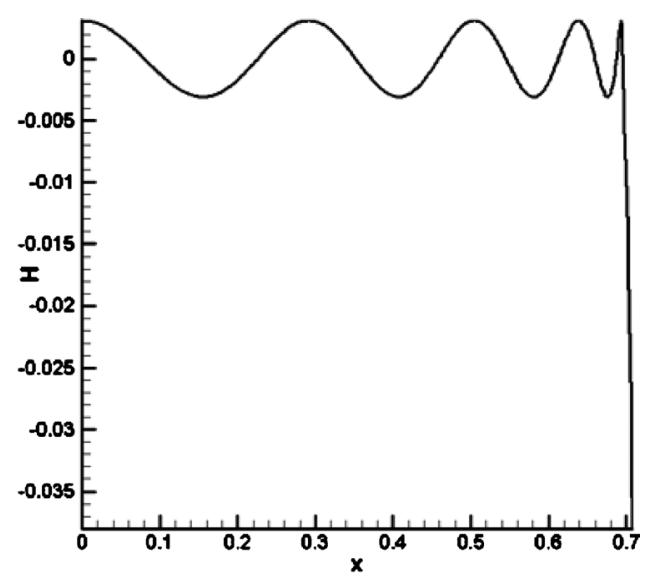

(b)

FIG. 10. Two snapshots of the threadline shape corresponding to the case with $\mathrm{R}=\rho_{\mathrm{g}} / \rho=0.00122, \mathrm{~L} / \mathrm{a}_{0}=100$, the $\operatorname{Reynolds}$ number $\mathrm{Re}=2 \mathrm{U}_{0} \mathrm{a}_{0} / \nu_{\mathrm{g}}=40$, $\mathrm{H}_{0 \omega}=0.01$, and the dimensionless perturbation frequency $\Omega=\omega \mathrm{L} / \mathrm{U}_{0}=0.01$ (for $\mathrm{L}=10 \mathrm{~cm}$ and $\mathrm{U}_{0}=10^{4} \mathrm{~cm} / \mathrm{s}, \omega=10 \mathrm{~Hz}$ ) at two different time moments. The perturbation amplitude $\mathrm{H}$ and the longitudinal coordinate $\mathrm{x}$ are rendered dimensionless by $\mathrm{L}$.

$$
\left.\mathrm{H}\right|_{\mathrm{x}=0}=\mathrm{H}_{0 \omega} \exp (\mathrm{i} \omega \mathrm{t}), \quad \partial \mathrm{H} /\left.\partial \mathrm{x}\right|_{\mathrm{x}=0}=0
$$

where $\mathrm{H}_{0 \omega}$ (which is a complex number in the general case) and real $\omega$ are the corresponding mode amplitude and frequency, and $\mathrm{i}$ is the imaginary unit.

The boundary conditions (10) fully determine the functions $\Phi(\cdot)$ and $\mathrm{F}(\cdot)$ in the "hyperbolic" part and reduce Eq. (9) to the following threadline configuration

$$
\mathrm{H}(\mathrm{x}, \mathrm{t})=\mathrm{H}_{0 \omega} \exp (\mathrm{i} \omega \mathrm{t}) \cos [\omega \mathrm{I}(\mathrm{x})] .
$$

The real function $\mathrm{I}(\mathrm{x})$ results from the evaluation of the integrals in Eq. (9) as

$$
\begin{aligned}
\mathrm{I}(\mathrm{x})= & \frac{2 \rho \pi \mathrm{a}_{0}^{2}}{\mathrm{q}_{\tau}}\left\{\left[\left(\frac{\mathrm{q}_{\tau} \mathrm{L}}{\pi \mathrm{a}_{0}^{2}}-\rho_{\mathrm{g}} \mathrm{U}_{0}^{2}\right) / \rho\right]^{1 / 2}-\left[\left(\frac{\mathrm{q}_{\tau}(\mathrm{L}-\mathrm{x})}{\pi \mathrm{a}_{0}^{2}}\right.\right.\right. \\
& \left.\left.\left.-\rho_{\mathrm{g}} \mathrm{U}_{0}^{2}\right) / \rho\right]^{1 / 2}\right\} .
\end{aligned}
$$

Note that

$$
\mathrm{I}(0)=0, \quad \mathrm{I}\left(\mathrm{x}_{*}\right)=\frac{2 \rho \pi \mathrm{a}_{0}^{2}}{\mathrm{q}_{\tau}}\left[\left(\frac{\mathrm{q}_{\tau} \mathrm{L}}{\pi \mathrm{a}_{0}^{2}}-\rho_{\mathrm{g}} \mathrm{U}_{0}^{2}\right) / \rho\right]^{1 / 2} .
$$

The physically relevant solution for the threadline shape in the "hyperbolic" part is given by the real part of Eq. (11).

On the other hand, in the "elliptic" part the characteristics are imaginary, and Eq. (9) reduces to

$$
\begin{aligned}
\mathrm{H}(\mathrm{x}, \mathrm{t})= & \exp (\mathrm{i} \omega \mathrm{t})\left\{\mathrm{A} \exp \left[-\mathrm{i} \omega \mathrm{I}\left(\mathrm{x}_{*}\right)\right] \exp [-\omega \mathrm{J}(\mathrm{x})]\right. \\
& \left.+\mathrm{B} \exp \left[\mathrm{i} \omega \mathrm{I}\left(\mathrm{x}_{*}\right)\right] \exp [\omega \mathrm{J}(\mathrm{x})]\right\},
\end{aligned}
$$

where $\mathrm{A}$ and $\mathrm{B}$ are constants and

$$
\mathrm{J}(\mathrm{x})=\frac{2 \rho \pi \mathrm{a}_{0}^{2}}{\mathrm{q}_{\tau}}\left[\frac{\mathrm{q}_{\tau} \mathrm{x}}{\rho \pi \mathrm{a}_{0}^{2}}-\frac{\mathrm{q}_{\tau} \mathrm{L} /\left(\pi \mathrm{a}_{0}^{2}\right)-\rho_{\mathrm{g}} \mathrm{U}_{0}^{2}}{\rho}\right]^{1 / 2} .
$$

Note that

$$
\mathrm{J}\left(\mathrm{x}_{*}\right)=0, \quad \mathrm{~J}(\mathrm{~L})=\frac{2 \rho \pi \mathrm{a}_{0}^{2}}{\mathrm{q}_{\tau}}\left(\frac{\rho_{\mathrm{g}} \mathrm{U}_{0}^{2}}{\rho}\right)^{1 / 2} .
$$

The constants are found via matching of Eq. (14) to Eq. (11) at $\mathrm{x}=\mathrm{x}_{*}$, which yields $\mathrm{A}=\mathrm{B}=\mathrm{H}_{0 \omega} / 2$. Then, the threadline shape in the "elliptic" part is given by

$$
\begin{aligned}
\mathrm{H}(\mathrm{x}, \mathrm{t})= & \mathrm{H}_{0 \omega} \exp (\mathrm{i} \omega \mathrm{t})\left\{\cosh [\omega \mathrm{J}(\mathrm{x})] \cos \left[\omega \mathrm{I}\left(\mathrm{x}_{*}\right)\right]\right. \\
& \left.+\mathrm{i} \sinh [\omega \mathrm{J}(\mathrm{x})] \sin \left[\omega \mathrm{I}\left(\mathrm{x}_{*}\right)\right]\right\} .
\end{aligned}
$$

As in the "hyperbolic" part, the physically relevant result for the "elliptic" part is given by the real part of Eq. (17). It is emphasized that since $\mathrm{dI} /\left.\mathrm{dx}\right|_{\mathrm{x}=\mathrm{x}_{*}}=\mathrm{dJ} /\left.\mathrm{dx}\right|_{\mathrm{X}=\mathrm{x}_{*}}=\infty$, the transition from the "hyperbolic" part to the "elliptic" part occurs in the cross-section $\mathrm{x}=\mathrm{x}_{*}$ where $\mathrm{dH} /\left.\mathrm{dx}\right|_{\mathrm{x}=\mathrm{x}_{*}}=\infty$, i.e. the threadline is oriented perpendicularly to the gas flow.

Two dimensionless snapshots of the threadline shape calculated using Eqs. (11), (12), (15), and (17) are plotted in Fig. 10. It is clearly seen that the wavelength of the standing perturbation wave in the "hyperbolic" part becomes shorter as approaching to the transition cross-section $\mathrm{x}=\mathrm{X}_{*}(=0.695$ in the present case). In the "elliptic" part after the transition the perturbation amplitude increases very rapidly, which in fact, rapidly violates the assumption of small perturbations. A fully realistic prediction of the threadline shape in this part can be achieved only in the framework of the fully nonlinear theory. However, a practical approximation is probably the one where the calculation is truncated at a cross-section $\mathrm{x}$ $>x_{*}$ where the perturbed threadline length reaches the value of the order of L (i.e., 1 in the dimensionless case).

The "explosive" behavior of the solution in the "elliptic" part is a clear manifestation of the fact that the elliptic problem is solved there as an initial value problem. This results from the matching condition imposed only at one side of the interval at the transition from the "hyperbolic" part at $\mathrm{x}=\mathrm{x}_{*}$. That means that in the "elliptic" part we are dealing with an Hadamard-like problem, which is inevitably prone to fast perturbation growth. Situations where physical problems reduce to the ill-posed Hadamard-like problems are not unique. For example, the well-known capillary Rayleigh instability, if treated in the quasi-one-dimensional, linearized approximation for small perturbations ${ }^{20}$ immediately reveals an 
Hadamard-like initial value problem for the Laplace equation with surface-tension-related regularization for the short waves.

Strong amplification of bending perturbation waves as they pass from the "hyperbolic" (stretched) to the "elliptic" (almost unstretched) part of the threadline is partially related to the fact that the wave brings with itself a parcel of mechanical energy delivered to it by turbulent eddies in the "initial" cross-section $\mathrm{x}=0$. In the almost unloaded part, this energy is released as kinetic energy of lateral excursions unrestricted by longitudinal stretching and manifesting themselves as flapping. Also, the distributed lift force strongly amplifies bending perturbations in the unloaded part of the threadline. This effect also significantly contributes to flapping.

In reality turbulent pulsations can affect threadline not only at the "initial" cross-section but over a certain length. Considering the effect of distributed turbulent pulsations on threadlines separately from the effect of the distributed lift force, we apply the formula for the oscillation magnitude $\left\langle\mathrm{A}_{\text {threadline }}\right\rangle$ derived in Sec. II accounting for the fact that the restrictive longitudinal tension diminishes in the $\mathrm{x}$ direction. Therefore, $\left\langle\mathrm{A}_{\text {threadline }}(\mathrm{x})\right\rangle=\left[2 \nu_{\mathrm{t}} \tau_{\mathrm{r}}(\mathrm{x})\right]^{1 / 2}$, where $\nu_{\mathrm{t}}$ $\left.=0.015 \mathrm{U}_{0} \mathrm{~d}_{0}, \quad \tau_{\mathrm{r}}(\mathrm{x})=\mathrm{L}\left[\sigma_{\mathrm{xx}}(\mathrm{x}) / \rho\right)\right]^{-1 / 2}$, whereas $\sigma_{\mathrm{xx}}(\mathrm{x})$ is given by Eqs. (1) and (3). As a result, we find the threadline envelope as

$$
\begin{aligned}
& \left\langle\mathrm{A}_{\text {threadline }}(\mathrm{x})\right\rangle \\
& \quad=0.16\left(\frac{\rho}{\rho_{\mathrm{g}}}\right)^{1 / 4}\left(\frac{\mathrm{U}_{0} \mathrm{~d}_{0}}{\nu_{\mathrm{g}}}\right)^{0.2025}\left(\frac{\mathrm{d}_{0}}{\mathrm{~L}}\right)^{1 / 4} \sqrt{\mathrm{d}_{0} \mathrm{~L}} \frac{1}{(1-\mathrm{x})^{1 / 4}},
\end{aligned}
$$

where $\mathrm{x}$ is rendered dimensionless by $\mathrm{L}$. The result shows that the shape of the threadline envelope should follow the dependence $\left\langle\mathrm{A}_{\text {threadline }}\right\rangle \sim(1-\mathrm{x})^{-1 / 4}$ if only turbulent pulsations are accounted for, whereas distributed aerodynamic lift force disregarded.

The theoretical predictions of this section are compared to the experimental data in Sec. IV.

\section{DISCUSSION: SOME THEORETICAL PREDICTIONS VERSUS EXPERIMENTAL DATA}

The results for longer threadlines in Fig. 7(a) show that the length of the flapping region $\mathrm{L}_{\mathrm{f}}$ does not vary with the threadline length L. This is in agreement with the theoretical prediction of Eq. (8).

The experimental data in Fig. 8 reveal the scaling $\mathrm{L}_{\mathrm{f}}$ $\sim \mathrm{U}_{\mathrm{g}}(0)$ at $\mathrm{U}_{\mathrm{g}}(0) \leq 230 \mathrm{~m} / \mathrm{s}$, which is close to the scaling $\mathrm{L}_{\mathrm{f}} \sim \mathrm{U}_{\mathrm{g}}(0)^{0.81}$ expected from Eq. (8). On the other hand, at $\mathrm{U}_{\mathrm{g}}(0)>230 \mathrm{~m} / \mathrm{s}$ the scaling changes to $\mathrm{L}_{\mathrm{f}} \sim \mathrm{U}_{\mathrm{g}}(0)^{2.42}$, which deviates significantly from the predictions of Eq. (8). A much stronger dependence of the aerodynamic drag on the gas jet velocity at such high values of $\mathrm{U}_{\mathrm{g}}(0)$ most probably corresponds to a strong increase in the eddy turbulent viscosity at higher blowing rates. The experiments in Ref. 19, which established the expression for the aerodynamic drag (1) used to derive Eq. (8) were conducted with threadlines moving in stagnant air, which does not involve high levels of

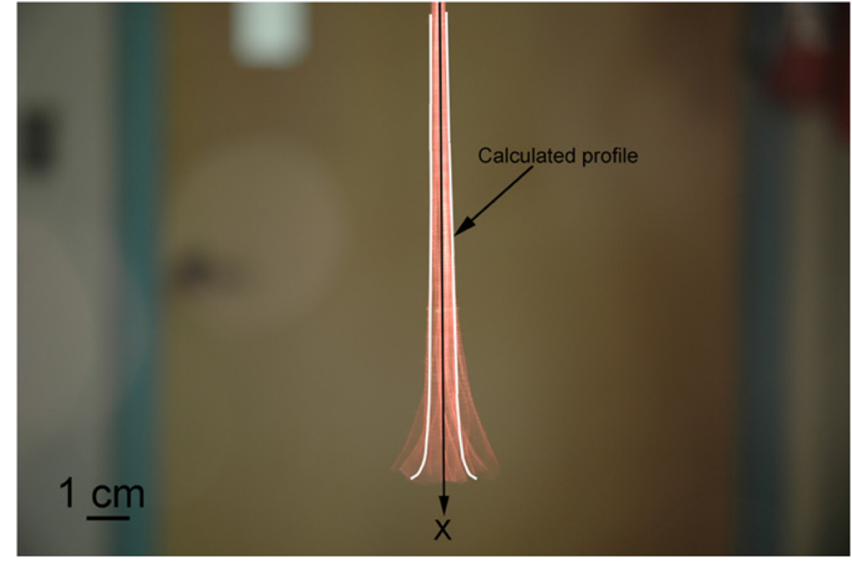

FIG. 11. (Color online) The envelope observed in the experiment with a 19 $\mathrm{cm}$ long threadline subjected to gas jet issued at 28 bar vs the prediction accounting for only the effect of turbulent pulsations and disregarding distributed aerodynamic lift force.

the eddy turbulent viscosity in blowing jets. Therefore, deviations from Eqs. (1) and (8) are thus expected at higher blowing velocities, and it is remarkable that these equations work rather accurately up to $230 \mathrm{~m} / \mathrm{s}$.

The comparison of the prediction of the effect of the distributed turbulent pulsations to the experimentally observed threadline envelope shown in Fig. 11 reveals the following. In the upper, hyperbolic part of the threadline where bending is strongly restricted by longitudinal stretching and the aerodynamic lift cannot be large, the envelope profile is rather accurately described by the predicted dependence of Eq. (18) $\left\langle\mathrm{A}_{\text {threadline }}\right\rangle \sim(1-\mathrm{x})^{-1 / 4}$. On the other hand, in the lower, elliptic part of the threadline the effect of turbulent pulsations alone is insufficient to describe the observed flapping amplitudes, which are mostly determined by the distributed aerodynamic lift force.

\section{v. CONCLUSION}

The experimental and theoretical study of threadline blowing in this work revealed the following mechanism of the process. Large turbulent eddies in the high speed gas jet with the eddy frequencies of the order of $10^{3} \mathrm{~Hz}$ impact the threadlines introducing bending perturbations. It takes multiple rapid eddy impacts on a massive threadline to cause a visible lateral excursion. The bending amplitude achieved locally by the eddy bombardment is controlled by a much slower (with the frequencies of about 10-100 Hz) propagation of bending perturbations along the stretched (by the aerodynamic drag) threadline. Bending perturbations propagate along the threadline and reach lower regions which are practically unstretched/unloaded, since they do not support the whole aerodynamic drag imposed on the threadline. There, the restrictions on large amplitude bending imposed by strong stretching are removed. Then, turbulence energy stored in the bending perturbations of the threadline can be released as the kinetic energy of a further bending. Moreover, bending perturbations of the unloaded threadlines are strongly amplified by the distributed aerodynamic lift force. As a result, the whole region close to the free unloaded threadline end experiences flapping of high amplitude. Blow- 
ing threadlines are capable of simultaneous extracting kinetic energy from both mean flow and turbulent pulsations in it.

\section{ACKNOWLEDGMENTS}

The current work is supported by the Nonwovens Cooperative Research Center (NCRC).

\section{APPENDIX: VELOCITY OF GAS FLOW AT THE NOZZLE EXIT}

Gas flows from a high pressure cylinder to open atmosphere (where it comes in contact with a threadline or polymer jet) through a long uniform pipe of cylindrical crosssection. Pressure drops are of the order of several tens of bars, i.e., we are dealing with an adiabatic compressible gas flow with friction. An approximate theory of such flows in uniform cylindrical pipes based on the compressible Bernoulli equation with friction can be found in Ref. 34 (p. 138) supplemented by standard isentropic formulae for gas flows. ${ }^{35}$ The theory reduces to the following three equations:

$$
\begin{aligned}
& \frac{1}{\lambda_{1}^{2}}-\frac{1}{\lambda_{2}^{2}}-2 \ln \left(\frac{\lambda_{2}}{\lambda_{1}}\right)=\mathrm{f} \frac{2 \kappa}{\kappa+1} \frac{\mathrm{L}}{\mathrm{d}_{0}}, \\
& \frac{\mathrm{p}_{01}}{\mathrm{p}_{2}}=\frac{\lambda_{2}}{\lambda_{1}} \frac{1}{\left[1-\lambda_{2}^{2}(\kappa-1) /(\kappa+1)\right]} \frac{1}{\left[1-\lambda_{1}^{2}(\kappa-1) /(\kappa+1)\right]^{1 /(\kappa-1)}},
\end{aligned}
$$

$$
\mathrm{u}_{2}=\left(\frac{2}{\kappa+1}\right)^{1 / 2} \lambda_{2} \mathrm{a}_{01}
$$

In Eqs. (A1)-(A3), $\lambda_{1}$ and $\lambda_{2}$ are the velocity coefficients at the pipe entrance and exit, respectively, which are both less than 1 in the subsonic flows we are dealing with, $\kappa$ is the ratio of the specific heat at constant pressure to the specific heat at constant volume, $\mathrm{L}$ and $\mathrm{d}_{0}$ are the pipe length and cross-sectional diameter, respectively, $\mathrm{f}$ is the friction factor, $\mathrm{p}_{01}$ is the stagnation pressure of gas in the cylinder, $\mathrm{p}_{2}$ is the atmospheric pressure, $\mathrm{u}_{2}$ is the gas velocity at the pipe exit, and $\mathrm{a}_{01}$ the adiabatic speed of sound in stagnant gas in the cylinder. For the friction factor in smooth pipes, the following expression (the Unwin formula) can be used: ${ }^{36}$

$$
\mathrm{f}=0.0025\left(1+3.6 / \mathrm{d}_{0}\right),
$$

where the pipe diameter $d_{0}$ is taken in inches. In the present case $\mathrm{d}_{0}=0.02^{\prime \prime}$ and thus $\mathrm{f}=0.45$.

Equations (A1) and (A2) define the velocity coefficient at the pipe exit $\lambda_{2}$ through the pressure ratio $\mathrm{p}_{01} / \mathrm{p}_{2}$ for any pipe length. After that Eq. (A3) is used to find the corresponding gas velocity $\mathrm{u}_{2}$. As the ratio $\mathrm{p}_{01} / \mathrm{p}_{2}$ increases, the value of $\lambda_{2}$ increases until $\lambda_{2}=1$ is inevitably reached at a certain value of $\left(\mathrm{p}_{01} / \mathrm{p}_{2}\right)_{\mathrm{c}}$, which corresponds to flow choking. At $\mathrm{p}_{01} / \mathrm{p}_{2}>\left(\mathrm{p}_{01} / \mathrm{p}_{2}\right)_{\mathrm{c}}$ velocity $\mathrm{u}_{2}$ does not change and is equal to $\mathrm{u}_{2 \mathrm{c}}=[2 /(\kappa+1)]^{1 / 2} \mathrm{a}_{01}$.

The predicted exit velocity of gas is plotted in Fig. 12 versus pressure ratio. The utmost right point of about $\mathrm{u}_{2}$ $=310.38 \mathrm{~m} / \mathrm{s}$ at $\mathrm{p}_{01} / \mathrm{p}_{2}=47.91$ corresponds to chocking. At the pressure ratios $\mathrm{p}_{01} / \mathrm{p}_{2}>47.91$ the exit velocity will stay the same $\mathrm{u}_{2}=310.38 \mathrm{~m} / \mathrm{s}$ due to chocking. The experimental data in Fig. 12 were acquired using two flow measuring de-

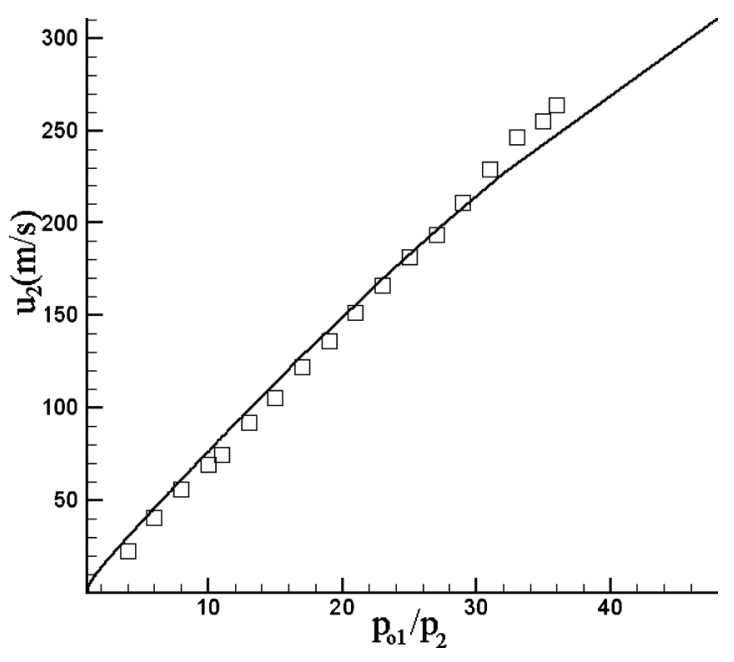

FIG. 12. Exit velocity vs pressure ratio for $\kappa=1.4, \mathrm{~L} / \mathrm{D}=3000$ and $\mathrm{a}_{01}$ $=340 \mathrm{~m} / \mathrm{s}$. The predicted velocity is shown by the curve, the experimental data-by symbols.

vices: one for the lower pressures $\mathrm{p}_{01} / \mathrm{p}_{2}<25$ with a higher accuracy (digital flow meter FMA-5610 from Omega), and another one for the higher pressures $\mathrm{p}_{01} / \mathrm{p}_{2}>25$ (Dwyer Instruments rotameter with a float; with a lower accuracy). The agreement of the predicted and measured velocities is very good practically in the whole range.

${ }^{1}$ R. R. Bresee, and W. C. Ko, Int. Nonwovens J. 12(2), 21 (2003).

${ }^{2}$ L. C. Pinchuk, V. A. Goldade, A. V. Makarevich, and V. N. Kestelman, Meltblowing, Equipment, Technology and Polymer Fibrous Materials (Springer, Berlin, 2002).

${ }^{3}$ M. A. J. Uyttendaele and R. L. Shambaugh, AIChE J. 36, 175 (1990).

${ }^{4}$ V. T. Marla and R. L. Shambaugh, Ind. Eng. Chem. Res. 42, 6993 (2003).

${ }^{5}$ V. T. Marla and R. L. Shambaugh, Ind. Eng. Chem. Res. 43, 2789 (2004).

${ }^{6}$ R. Chhabra and R. L. Shambaugh, Int. Nonwovens J. 13(3), 24 (2004).

${ }^{7}$ N. Marheineke and R. Wegener, SIAM J. Appl. Math. 66, 1703 (2006).

${ }^{8}$ N. Marheineke and R. Wegener, SIAM J. Appl. Math. 68, 1 (2007).

${ }^{9}$ T. Götz, A. Klar, N. Marheineke, and R. Wegener, SIAM J. Appl. Math. 67, 1704 (2007)

${ }^{10}$ L. L. Bonilla, T. Götz, A. Klar, N. Marheineke, and R. Wegener, SIAM J. Appl. Math. 68, 648 (2007).

${ }^{11}$ M. P. Paidoussis, J. Fluid Mech. 26, 717 (1966).

${ }^{12}$ G. S. Triantafyllou, Phys. Fluids A 4, 544 (1992).

${ }^{13}$ M. Shelley, N. Vandenberghe, and J. Zhang, Phys. Rev. Lett. 94, 094302 (2005).

${ }^{14}$ D. J. J. Farnell, T. David, and D. C. Barton, J. Fluids Struct. 19, 29 (2004).

${ }^{15}$ M. Argentina and L. Mahadevan, Proc. Natl. Acad. Sci. U.S.A. 102, 1829 (2005).

${ }^{16}$ C. H. K. Williamson and R. Govardhan, Annu. Rev. Fluid Mech. 36, 413 (2004).

${ }^{17}$ B. S. H. Connell and D. K. P. Yue, J. Fluid Mech. 581, 33 (2007).

${ }^{18}$ A. Ziabicki, Fundamentals of Fibre Formation (Wiley, New York, 1976).

${ }^{19}$ High-Speed Fiber Spinning, edited by A. Ziabicki and H. Kawai (Wiley, New York, 1985).

${ }^{20}$ A. L. Yarin, Free Liquid Jets and Films: Hydrodynamics and Rheology (Longman, Harlow and Wiley, New York, 1993).

${ }^{21}$ C. Weber, Z. Angew. Math. Mech. 11, 136 (1931).

${ }^{22}$ P. Debye and J. Daen, Phys. Fluids 2, 416 (1959).

${ }^{23}$ V. M. Entov and A. L. Yarin, J. Fluid Mech. 140, 91 (1984).

${ }^{24}$ D. H. Reneker, A. L. Yarin, H. Fong, and S. Koombhongse, J. Appl. Phys. 87, $4531(2000)$.

${ }^{25}$ A. L. Yarin, S. Koombhongse, and D. H. Reneker, J. Appl. Phys. 89, 3018 (2001).

${ }^{26}$ D. H. Reneker, A. L. Yarin, E. Zussman, and H. Xu, Adv. Appl. Mech. 41, 43 (2007).

${ }^{27}$ S. I. Pai, Fluid Dynamics of Jets (Van Nostrand Company, Macmillan, Toronto, London, 1954). 
${ }^{28}$ G. N. Abramovich, The Theory of Turbulent Jets (MIT, Boston, 1963).

${ }^{29}$ S. B. Pope, Turbulent Flows (Cambridge University Press, Cambridge, 2000).

${ }^{30}$ A. L. Yarin, S. Sinha-Ray, and B. Pourdeyhimi, J. Appl. Phys. 108, 034913 (2010).

${ }^{31}$ R. A. Antonia, B. R. Satyaprakash, and A. K. M. F. Hussain, Phys. Fluids 23, 695 (1980)

${ }^{32}$ Handbook of Turbulence, edited by W. Frost and T. H. Moulden (Plenum, New York, 1977), Vol. 1
${ }^{33}$ A. L. Yarin, in Springer Handbook of Experimental Fluid Mechanics edited by C. Tropea, A. L. Yarin, and J. Foss (Springer, Berlin, 2007), pp. 57-82.

${ }^{34}$ W. L. McCabe, J. C. Smith, and P. Harriott, Unit Operations in Chemical Engineering 5th ed. (McGraw-Hill, New York, 1993).

${ }^{35}$ L. G. Loitsyanskii, Mechanics of Liquids and Gases (Pergamon, Oxford, 1966).

${ }^{36}$ E. S. Menon, Piping Calculations Manual (McGraw-Hill, New York, 2005). 